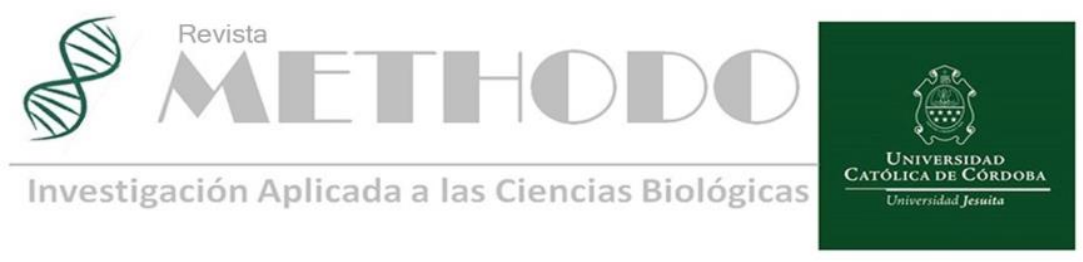

CASO CLINICO Rev. Methodo 2021;6(4):190-193

https://doi.org/10.22529/me.2021.6(4)07

Recibido 14 Jul. 2021 | Aceptado 30 Ago. 2021|Publicado 05 Oct. 2021

\title{
Roséola, alopecia y amigdalitis como manifestación de secundarismo sifilítico
}

\section{Roseola, alopecia and tonsilitis as a manifestation of syphilitic secondarism}

\author{
Sofía Carla Juárez ${ }^{1}$ (D) , Ana Laura Gallmann ${ }^{1}$, Julieta Roxana Brusa ${ }^{1}$, María Noelia Andrade ${ }^{1}$, María \\ Jimena Nocito ${ }^{1}$, Mariana Beatriz del Valle Papa ${ }^{1}$ \\ 1. Universidad Católica de Córdoba, Facultad de Ciencias de la Salud. Clínica Universitaria Reina Fabiola. Servicio de Dermatología \\ Correspondencia: Sofía C. Juárez. E-mail: juarez.sofiac@gmail.com.
}

\section{Resumen}

La sífilis es una de las enfermedades infectocontagiosas de transmisión sexual más frecuentes, que presenta diferentes estadios en su evolución clínica. El estadio secundario se caracteriza por un gran polimorfismo lesional, que compromete la piel, las mucosas y los anexos cutáneos, motivo por el cual se la conoce como "la gran simuladora". Es importante el conocimiento de todas sus manifestaciones para su sospecha, diagnóstico y oportuno tratamiento.

Palabras clave: Sífilis. Lúes. Roséola. Alopecia. Amigdalitis.

\section{Abstract}

Syphilis is one of the most common sexually transmitted infectious diseases, which presents different stages in its clinical course. The secondary stage is characterized by a large lesional polymorphism, which involves the skin, mucous membranes and cutaneous appendages, which is why it is known as "the great simulator". Knowledge of all its manifestations is important for its possible identification, diagnosis and subsequent treatment.

Keywords: Syphilis. Lúes. Roseola. Alopecia. Tonsillitis.

\section{Introducción}

La sífilis es una enfermedad infectocontagiosa, sistémica y crónica, causada por Treponema pallidum subespecie pallidum $^{1-3}$. Se transmite principalmente de manera sexual, a través de la piel, mucosas o semimucosas en contacto con un individuo infectado con lesiones correspondientes a estadios primarios o secundarios de la enfermedad, aunque también puede adquirirse de manera congénita, vía transplacentaria o por el canal del parto durante el nacimiento ${ }^{1,2,4}$. El interés de esta enfermedad se debe a la heterogeneidad de manifestaciones mucocutáneas, pocas veces reconocidas por médicos poco avezados, lo que dificulta el diagnóstico precoz, con el consecuente riesgo de transmisión a otras personas y evolución de la enfermedad hacia estadios evolutivos de mayor gravedad ${ }^{1,3,4-6}$. 


\section{Caso clínico}

Presentamos el caso de un paciente de sexo masculino de 24 años de edad, sin antecedentes personales patológicos, alérgico a la penicilina. Se presentó a la consulta dermatológica por pérdida del cabello de un mes de evolución. Al examen físico dermatológico, se observaron placas alopécicas, en forma de parches, de aspecto apolillado, con bordes pocos definidos y pelos cortados de diferentes longitudes, sin signos inflamatorios, localizados en región temporooccipital. (Figura 1a y 1b)

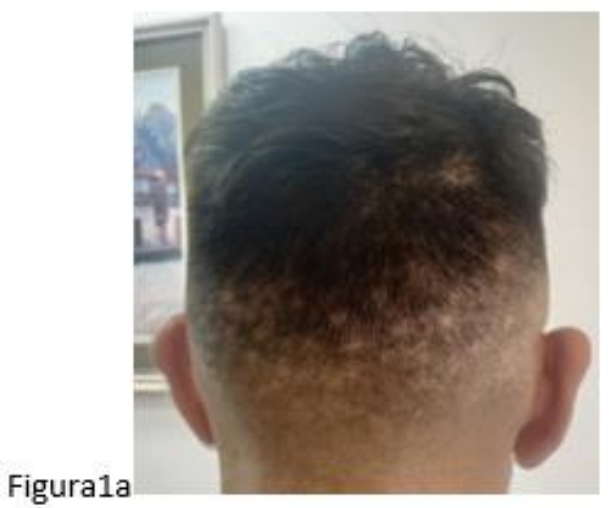

Figura $1 \mathrm{~b}$

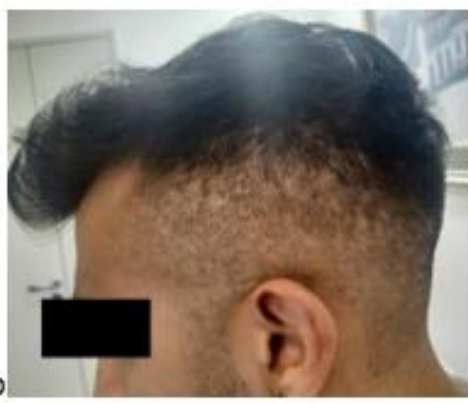

Figura 1. Alopecia en patrón apolillado de localización a) occipital b) temporal.

Al realizar un análisis retrospectivo de su evolución clínica, el paciente consultó tres meses previos por guardia central por lesiones clínicamente compatibles con roséola sifilítica, pero fue interpretado por el médico como picadura de insectos y medicado con antihistamínicos, con resolución de las mismas a los pocos días. Posteriormente fue valorado por Servicio de Clínica Médica y derivado a Otorrinolaringología por odinofagia de un mes de evolución. En ese momento, se evidenció una mucosa faríngea eritematosa, congestiva y amígdala derecha indurada, con placa blanquecina semitransparente que recubría la totalidad de la misma y se desprendía en bloque al removerla con hisopo, asociado a adenopatías cervicales móviles. (Figura 2) Presentó un análisis de laboratorio con citológico, proteína $\mathrm{C}$ reactiva y eritrosedimentación, con resultados dentro de parámetros normales. Un hisopado faríngeo para Streptococcus beta hemolítico del grupo A y monotest para mononucleosis infecciosa, negativos. Además, se le realizó tomografía computada de cuello que descartó la presencia de colecciones y se tomó biopsia de amígdala cuyo estudio histopatológico informó la presencia de infiltrado inflamatorio mixto inespecífico.

En nuestra consulta dermatológica, teniendo en cuenta el patrón de alopecia más el antecedente de amigdalitis y de la probable roséola sifilítica no reconocida en guardia central, se sospechó el diagnóstico de sífilis secundaria y se solicitó VDRL (Venereal Disase Research Laboratory), al cual se agregó también serologías virales (HIV, hepatitis $\mathrm{B}$ y C). Los resultados fueron negativos, excepto el hallazgo de una VDRL cuantitativa de 1:32, confirmando la sospecha diagnóstica. Se inició tratamiento con doxiciclina $100 \mathrm{mg}$ cada 12 horas por 28 días. Se realizaron pruebas treponémicas (FTA-abs: Fluorescent Treponemal Antibody-Absorption) cuyo resultado informó IgG para Treponema pallidum positivo. Se controló al paciente intratratamiento, con evolución favorable, pero posteriormente no regresó para su seguimiento.

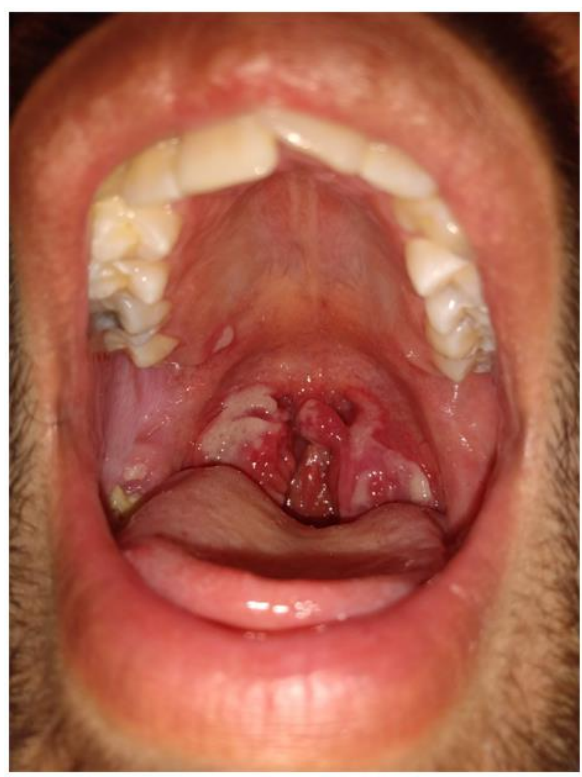

Figura 2. Amígdala derecha con placa blanquecina semitransparente, y pequeña ulcera cubierta por membrana blanquecina en paladar duro.

\section{Discusión}

La sífilis es una de las infecciones de trasmisión sexual más frecuente, cuya incidencia continua en aumento $^{3}$. En Argentina, donde es una enfermedad de notificación obligatoria, los contagios continúan creciendo sostenidamente desde el año 2010, con una

Revista Methodo: Investigación Aplicada a las Ciencias Biológicas. Universidad Católica de Córdoba. Jacinto Ríos 571 Bo Gral. Paz. X5004FXS. Córdoba. Argentina. Tel.: (54) 3514517299 / Correo: methodo@ucc.edu.ar / Web: methodo.ucc.edu.ar | CASO CLINICO Rev. Methodo 2021;6(4):190-193. 
incidencia reportada en el 2018 de 51,1 casos por cada 100000 habitantes s $^{3,5,7}$.

La evolución natural de la sífilis tiende a la cronicidad, con períodos de latencia y exacerbaciones, pasando por diferentes estadios sucesivos $^{1}$. Luego de un período de incubación de 3 semanas (entre 10 a 90 días), aparece la lesión primaria o chancro, ubicado en el sitio de inoculación del Treponema, genital o extragenital. El mismo, que generalmente se acompaña de linfadenopatía satélite regional, generalmente único e indoloro, cura espontáneamente entre los 30 y 45 días. En ocasiones es múltiple y doloroso, cuando es de etiología mixta o se encuentra sobreinfectado. Posteriormente, se produce la diseminación sanguínea del microorganismo, y alrededor de los 60 días, comienzan manifestaciones del estadio secundario, las cuales pueden comprometer piel, mucosas, faneras y estar acompañadas o no de linfadenopatías generalizadas y síntomas constitucionales. Las lesiones son superficiales, no destructivas y curan espontáneamente, alternando así, períodos de latencia asintomáticos y recidivas, que duran años. Se puede decir que, epidemiológicamente, los estadios tempranos de la infección son los que más importan, ya que son de mayor contagiosidad ${ }^{3}$. Finalmente, el estadio terciario de la enfermedad se manifiesta sólo en un tercio de pacientes que no reciben tratamiento ${ }^{1,3-6,8}$.

El gran espectro de manifestaciones clínicas del estadio secundario sifilítico, hace que se la conozca a la misma como "la gran simuladora". Sin embargo, muchas de ellas, son de presentación poco frecuentes, lo que dificulta su sospecha, en especial cuando aparecen de manera aislada ${ }^{1,3}$.

El compromiso cutáneo suele ser polimorfo. Inicialmente puede aparecer un exantema macular evanescente asintomático, llamado roséola sifilítica, a menudo no reconocido como ocurrió en nuestro paciente. Posteriormente pueden aparecer pápulas eritematosas, infiltradas, asintomáticas (sifílides papulosas), rodeadas por un reborde descamativo llamado "collarete de Biet". Otras formas de sifílides pueden ser liquenoides, micropapulares, foliculares, vesiculares, corimbiformes, psoriasiformes, nodulares, anulares, nóduloulcerativa, papuloescamosa fotodistribuida, tipo linfoma $\mathrm{y}$ pustulosa. Curan sin dejar cicatriz, aunque pueden aparecer cambios pigmentarios residuales conocidos como sifílides pigmentarias ${ }^{3,8}$.

Las lesiones mucosas sifilíticas se presentan sólo en un $30 \%$ de los pacientes y rara vez son la única manifestación de la enfermedad ${ }^{4,9,10}$. Si bien la mucosa oral puede comprometerse en cualquier estadio, en el secundario es frecuente encontrar exulceraciones asintomáticas, lesiones blanco nacaradas definidas, conocidas como sifílides opalinas, lesiones papulo-hipertróficas, vegetantes, eritematosas o eritemato-grisáceas llamadas condilomas planos. En la lengua, placas eritematosas depapiladas (sifílides en pradera segada) y en comisura bucal, pápulas divididas o fisuradas. Puede comprometerse también la faringe, con la consecuente faringitis y amigdalitis, como presentó nuestro paciente. Existen pocos casos reportados en la literatura de esta última manifestación, en la cual es característico encontrar amígdalas edematosas, congestivas, cubiertas de exudado fino, color grisáceo, que puede ser confundida con amigdalitis por streptococcus, virales (VEB, adenovirus, coxsackie), tuberculosis, angina de Vincent, carcinoma epidermoide o linfomas ${ }^{4,9,10}$. De realizarse una biopsia para su estudio, los cambios histopatológicos incluyen infiltración perivascular con células plasmáticas y linfocitos e hiperplasia reactiva. Debido a que espiroquetas son saprófitas de cavidad oral, no se deben realizar técnicas de campo oscuro para visualizar Treponemas, pero si puede ser identificados mediante técnica de inmunohistoquímica ${ }^{2,9,10}$.

Con respecto al compromiso de anexos, la alopecia sifilítica es una manifestación infrecuente de la enfermedad, que se da principalmente durante el estadio secundario entre un 2,9 y $7 \%$ de los $\operatorname{casos}^{11-}$ ${ }^{13}$. Ésta puede afectar no sólo el cuero cabelludo, sino también otras áreas pilosas del cuerpo, como cejas, pestañas, barba y miembros ${ }^{3,11,12}$. Aunque su patogenia se desconoce, se estima que podría generarse por una respuesta inflamatoria al Treponema pallidum, o bien, por la simple presencia de este microorganismo que causaría la detención y anomalías de crecimiento del cabello, pérdida de pelos terminales con folículos vacíos y pelos $\operatorname{rotos}^{13}$. Las características clínicas, tricoscópicas e histopatológicas, pueden ayudar a distinguir la alopecia sifilítica de otros tipos de alopecias13. Además, se conocen diferentes patrones de presentación. El patrón apolillado o en transquilones, como presentó nuestro paciente, es el más frecuente y se lo considera un signo patognomónico de sífilis ${ }^{11,13}$. Se caracteriza por la presencia de múltiples áreas alopécicas, de diferentes diámetros y bordes pocos definidos, que se localizan principalmente en la región temporo-occipital, zona del cuero cabelludo más vascularizada y por lo tanto con mayor cantidad de treponemas. Muchas veces este tipo de alopecia es confundida con alopecia areata, tricotilomania o tiña capitis $^{12}$. Por otro lado, el patrón difuso se caracteriza por un compromiso generalizado, similar a un efluvio telógeno, y en contraste, este patrón puede ser además una forma de presentación de una reacción de JarishHerxheimer tras iniciar el tratamiento para la sífilis ${ }^{12}$. En algunos pacientes pueden coexistir ambos patrones, llamándose patrón mixto ${ }^{11,12}$. Se encuentra descripta, además, la presencia de tricodinia asociada a alopecia, que generalmente se autolimita días posteriores a iniciado el tratamiento de la enfermedad ${ }^{11}$.

Cuando se realiza tricoscopía de áreas alopécicas se pueden encontrar una reducción de pelos terminales y áreas foliculares sin pelos o vellos emergentes, con ausencia de puntos amarillos y negros. Es característico la coloración del cuero cabelludo

Revista Methodo: Investigación Aplicada a las Ciencias Biológicas. Universidad Católica de Córdoba

Jacinto Ríos 571 Bo Gral. Paz. X5004FXS. Córdoba. Argentina. Tel.: (54) 3514517299 / Correo:

methodo@ucc.edu.ar / Web: methodo.ucc.edu.ar | CASO CLINICO Rev. Methodo 2021;6(4):190-193. 
eritemato-amarronado en estas zonas, la cual desaparece posterior al tratamiento de la enfermedad ${ }^{11}$.

La toma de biopsia cutánea en zonas alopécicas no siempre está indicada. En su estudio histopatológico podemos encontrar una epidermis conservada con zonas de hiperqueratosis folicular, infiltrado dérmico linfohistiocitario perivascular y perifolicular con células plasmáticas. Además, se ha podido identificar la presencia de Treponema pallidum en folículos afectados gracias a estudios moleculares y técnicas de inmunohistoquímico ${ }^{12,14}$.

El estudio de lesiones en piel, mucosa oral y anexos cutáneos deben completarse con serologías que confirmen el diagnóstico.

Como conclusión, destacamos el compromiso médico en conocer las múltiples y variadas manifestaciones de la enfermedad, para poder sospecharlas, reconocerlas, realizar un diagnóstico y tratamiento precoz y oportuno de una enfermedad infectocontagiosa curable, que registra aumento de su incidencia. Es importante remarcar la importancia del examen completo del paciente, sin olvidar los anexos cutáneos y las regiones mucosas.

\section{Bibliografía}

1. Carrada Bravo T. Sífilis: actualidad, diagnóstico y tratamiento. Rev Fac Med UNAM 2003; 46 (6): 236-242.

2. Stary G, Stary A. Capítulo 82 Infecciones de transmisión sexual. Bolognia JL, Schaffer JV, Cerroni L. Dermatología. Vol 2. $4^{\circ}$ Edición. España: El Sevier; 2019

3. Bermejo A, Leiro V, Colasanti M, Solís Ramírez M ét al. Sífilis secundaria, 590 dilemas resueltos. Med Cutan Iber Lat Am. 2020; 48 (1): 21-28 doi:10.35366/93976.

4. Çakmak SK, Tamer E, Karadağ AS, Waugh M. Syphilis: A great imitator. Clinics in Dermatology. 2019; 37: 182-191 doi: 10.1016/j.clindermatol.2019.01.007.

5. Ivars Lleóa ME, Clavo Escribano P, Menéndez Prietoc B. Manifestaciones cutáneas atipícas en la sífilis. Actas Dermosifiliogr. 2015; 107: 275-283 doi: 10.1016/j.ad.2015.11.002

6. Bermejo A, Leiro V. Sífilis. El desafío permanente. Dermatol. Argent 2011; 17: 156159

7. Boletín sobre el VIH, sida e ITS en la Argentina. N³6. Año XXII-2019
8. Gatti JC, Cardama JE. Enfermedades de transmisión sexual (ETS). Manual de Dermatología. $11^{\circ}$ Edición. Buenos Aires, Argentina. Librería "El Ateneo" editorial. 1989; 185-211

9. Leuci S, Martina S, Adamo D, Ruoppo E ét al. Oral Syphilis: a retrospective analysis of 12 cases and a review of the literature. Oral Dis. 2013; 19: 738-46 doi: 10.1111 / odi.12058.

10. Hemlyn E, Marriott D, Gallagher RM. Secondary syphilis presenting as tonsillitis in three patients. The Journal of Laryngology \& Otology. 2006; 12: 602-604. doi: 10.1017 / S002221510600096X.

11. Piraccini BM, Broccoli A, Starace M, Gaspari $\mathrm{V}$ ét al. Hair and Scalp Manifestations in Secondary Syphilis: Epidemiology, Clinical Features and Trichoscopy. Dermatology. 2015; 231: 171-6. doi: 10.1159 / 000431314.

12. Hernández Bel $\mathrm{P}$, Unamuno $\mathrm{B}$, Sánchez Carazo JL, Febrer I ét al. Alopecia sifilítica: presentación de 5 casos y revisión de la literatura. Actas Dermosifiliogr. 2013; 104: 512-517. doi: 10.1016/j.ad.2012.02.009

13. Doche I, Hordinsky MK, Valente NYS, Romiti R ét al. Syphilitic Alopecia: Case Reports and Trichoscopic Findings. Skin Appendage Disord. 2017; 3: 222-224. doi.org/10.1159/000477415

14. Francois Jordaan H, Louw M. The moth-eaten alopecia of secondary syphilis. The American Journal of Dermatopathology. 1995; 17: 158162. doi: 10.1097 / 00000372-19950400000008 .

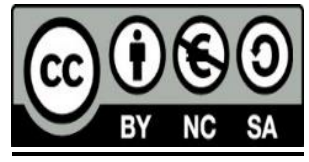

